Diabetologe 2014 · 10:443-444

DOI 10.1007/s11428-014-1262-0

Online publiziert: 19. September 2014

(c) Springer-Verlag Berlin Heidelberg 2014

W.A. Scherbaum ${ }^{1} \cdot$ S. Martin ${ }^{2} \cdot$ R. Landgraf ${ }^{3} \cdot$ L. Heinemann ${ }^{4}$. Im Namen der Vertreter deutschsprachiger diabetologischer Fachjournale

${ }^{1}$ Universitätsklinikum Düsseldorf, Heinrich-Heine-Universität Düsseldorf

${ }^{2}$ Westdeutsches Diabetes und Gesundheitszentrum, Düsseldorf

${ }^{3}$ Deutsche Diabetes Stiftung, München

${ }^{4}$ Profil Institut für Stoffwechselforschung, Neuss

\title{
Sichtbarkeit der deutschen Diabetologie
}

und in den deutschsprachigen Nachbarländern gibt, die potenziell ein Interesse haben, in diesen deutschsprachigen Journalen zu publizieren. Dabei ist der Begriff Diabetologe weit gefasst; dies können sehr wohl auch ärztliche Kollegen aus anderen Fachbereichen oder Wissenschaftler sein, die sich mit Themen beschäftigen, die für die Diabetologie in irgendeiner Form von Relevanz sind. Unsere Wahrnehmung ist, dass die Zahl dieser Kollegen und die der auf diesem Gebiet wissenschaftlich aktiven Zentren eher abnehmen. An dieser Entwicklung kann dieses Editorial nicht unbedingt etwas ändern. Es ist vielmehr eine politische Aufgabe, dies zu ändern. Wie ist das gemeint? Uns geht es darum, die Konsequenzen zu benennen: Es gibt weniger relevante deutschsprachige Publikationen.

Die Frage ist, warum sollte ein Publikationswilliger in einer deutschsprachigen Zeitschrift publizieren? Der Aufwand, hier ein Manuskript zu verfassen, dieses erfolgreich durch den Review-Prozess zu bekommen und letztendlich zu veröffentlichen, unterscheidet sich nicht wesentlich von dem Aufwand, den die Autoren für eine englischsprachige Publikation aufwenden müssen. Eine Publikation in englischer Sprache bietet aber einen potenziell größeren Leserkreis, und die Arbeit erhält mehr nationale und internationale Beachtung. Dabei ist aber zu bedenken, dass zahlreiche wissenschaftliche Arbeiten, die in einem nationalen Kontext höchst relevant sind, durch die Reviewer internationaler Journale oft nicht entsprechend ge- würdigt und abgelehnt werden. Viele englischsprachige Zeitschriften haben einen höheren „impact factor“. Da die Qualität der wissenschaftlichen Arbeit eines Wissenschaftlers überwiegend durch solche Indizes bemessen wird, insbesondere im universitären Bereich und in Forschungsinstituten, halten viele Leiter von diabetologischen Abteilungen ihre Mitarbeiter dazu an, unbedingt in englischsprachigen Zeitschriften zu publizieren. Diese Haltung ist im individuellen Fall nachvollziehbar, führt aber - insgesamt betrachtet - dazu, dass deutschsprachig publizierte Arbeiten generell unterbewertet werden und international nicht wahrgenommen werden. Dieses Defizit wird auch dadurch meist nicht behoben, dass die Abstracts deutschsprachiger Publikationen in Englisch übersetzt werden. Diese auch andere Sprachräume betreffende Tendenz führt dazu, dass, wie es Professor Jürgen Trabant in einem kürzlich publizierten Artikel in der Frankfurter Allgemeinen Zeitung ausdrückte, „durch die Anglophonie in der Wissenschaft ganze Bibliotheken von Wissen auf Nimmerwiedersehen verschwinden“. Es würde nicht nur eines Umdenkens in der akademischen Welt und der Bewertung von wissenschaftlichem Erfolg bedürfen, sondern möglicherweise auch Änderungen bei Habilitationsordnungen etc. erfordern, damit deutschsprachige Publikationen wieder eine angemessene Würdigung erfahren und es für Autoren sinnvoll, notwendig und attraktiv ist, in einem deutschsprachigen diabetologischen Jour- 
nal zu publizieren. Viele deutsche Diabetologen wissen gar nicht, welch hochkarätige Forschung in deutschen Kliniken, Instituten und Versorgungszentren geleistet wird. Die Zentren müssten sich aber in diesem Wissenstransfer deutlich besser einbringen - deutschsprachige Publikationen sind auch in diesem Sinne hilfreich.

Man kann natürlich provokant die Frage stellen: Brauchen wir überhaupt Publikationen in deutscher Sprache? Da viele Kollegen und ihre Mitarbeiter gerade in der klinischen Versorgung es nicht gewohnt sind, englische Texte zu lesen, bedeutet der Wegfall von deutschsprachigen Publikationen den Verlust von wesentlichen Informationen. Es würde den wissenschaftlichen und klinischen Austausch und die Ausbildung der Kollegen hierzulande eindeutig befördern, wenn gerade Studienergebnisse zu Epidemiologie, Versorgung und Gesundheitsökonomie, um nur ein paar Beispiele zu nennen, in deutscher Sprache und im Bezug zu unserem Gesundheitssystem publiziert werden würden. Die Publikation solcher Ergebnisse in einem nationalen Forum ist bisweilen wertvoller als die Publikation in einer internationalen Zeitschrift mit anderen Schwerpunkten.

In diesem Zusammenhang gilt es, ein weiteres Problem anzusprechen: die adäquate Begutachtung von eingereichten Manuskripten. Ziel des Review-Prozesses ist es, durch unabhängige externe Gutachter sicherzustellen, dass die Inhalte des Manuskripts korrekt sind und dem aktuellen Wissenstand entsprechen. Eine Begutachtung mit konstruktiver Kritik und Verbesserungs- und Ergänzungsempfehlungen ist für die Qualität der Manuskripte in den meisten Fällen ausgesprochen wichtig. Die Zeit, die die Gutachter aufwenden, erfährt jedoch keinerlei Würdigung, d. h., die Stunden, die hierfür benötigt werden, müssen unter akademische Aufgaben und Ehrenamtlichkeit verbucht werden. Dafür interessiert sich kaum eine Forschungseinrichtung oder Klinikleitung, d. h., die Gutachter investieren ihre Freizeit. Im Zusammenhang mit der schon angesprochenen Situation der weniger werdenden klinischen Diabeteszentren in Deutschland scheint auch die Anzahl von Kollegen weniger zu werden, die fähig und willig sind, diese Aufgabe wiederholt auf sich zu nehmen. Wir haben es vielleicht versäumt, die Bedeutung dieser Arbeit entsprechend anzuerkennen und ihr den notwendigen Platz einzuräumen.

Wir hoffen, durch dieses gemeinsame Editorial eine Diskussion zu initiieren, die im Endeffekt dazu führt, dass die deutsche Diabetologie sichtbar bleibt oder sichtbarer wird - und zwar durch das Beste, was man sich vorstellen kann: durch exzellente deutschsprachige Publikationen aus Forschung, Klinik und Patientenbetreuung.

\section{Für die Zeitschriften}

\author{
Der Diabetologe \\ Prof. Dr. Werner A. Scherbaum \\ Prof. Dr. Stefan Martin \\ Prof Dr. Rüdiger Landgraf
}

Diabetes und Stoffwechsel

Prof. Dr. Robert Ritzel

Prof. Dr. Karsten Müssig

Prof. Dr. Monika Kellerer

Prof. Dr. Dirk Müller-Wieland

Prof. Dr. Andreas Fritsche

Prof. Dr. Baptist Gallwitz

Diabetes, Stoffwechsel und Herz

Prof. Dr. Diethelm Tschöpe

Prof. Dr. Oliver Schnell

Prof. Dr. Christian A. Schneider

\section{Diabetes Congress Report \\ Prof. Dr. Reingard Bretzel \\ Prof. Dr. Hans Janka \\ Prof. Dr. Lutz Heinemann}

\section{Korrespondenzadresse}

Prof. Dr. W.A. Scherbaum

Heinrich-Heine-Universität,

Universitätsklinikum Düsseldorf

Moorenstr. 5, 40225 Düsseldorf

scherbaum@uni-duesseldorf.de

\section{Die Highlights vom DGIM-Kongress 2014 online!}

Immer gut informiert: Online fortbilden mit den neuen e.Lectures der DGIM | e.Akademie!

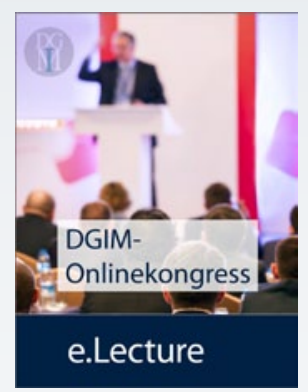

- Internistisches Fachwissen für die Praxis

- Jeder Onlinekurs mit

- kurzen Einstiegsfragen

- Vortragsvideo (ca. 15 min)

- Präsentation zum Vortrag

- MC-Fragen-Quiz
Alle Kurse exklusiv und kostenfrei für DGIM-Mitglieder:

DPSA-Screening - „state of the art"

- Metabolischer Stress und Ernährungsverhalten

- Rationale Therapie der schweren Pankreatitis

- Palliativmedizin bei Bedarf?

- Differenzialdiagnose chronischer Diarrhö

www.springermedizin.de/

internistenkongress 